\title{
ENERGY-BASED PHARMACOPHORE MODELING, VIRTUAL SCREENING, AND MOLECULAR DYNAMICS TO IDENTIFY POTENTIAL INHIBITORS FOR GLYCOGEN SYNTHASE KINASE 3 BETA
}

\author{
SHEEMA JB, WAHEETA HOPPER* \\ Department of Biotechnology, Faculty of Engineering and Technology, SRM University, Kattankulathur, Chennai - 603 203, \\ Tamil Nadu, India. Email: srmbioinformaticsresearch@gmail.com
}

Received: 07 October 2017, Revised and Accepted: 08 November 2017

\section{ABSTRACT}

Objective: Glycogen synthase kinase 3 beta (GSK3 $\beta$ ) is one of the main targets for wound healing activity. Our objective is to identify novel inhibitors for GSK3 $\beta$ using in silico approach.

Methods: Grid-based molecular docking, energy-based pharmacophore (e-pharmacophore) modeling, and molecular dynamics (MD) studies were performed for phytocompounds with GSK3 $\beta$ and compared with standard drugs using Schrodinger software.

Results: The glide scores and the molecular interactions of the phytocompounds were well comparable to the standard drugs. The MD was performed for the target bound to the best scoring ligand, entagenic acid. The pharmacophore features of this docked complex were modeled as e-pharmacophore. The constructed e-pharmacophore model was screened against phytocompounds retrieved from literature to identify the ligands with similar pharmacophore features.

Conclusion: The glide scores of fukinolic acid, cimicifugic acid, and linarin were $-10.99,-8.28$, and $-7.25 \mathrm{kcal} / \mathrm{mol}$, respectively. The further 50 nanoseconds MD study determined the stability of GSK3 $\beta$-linarin complex. Nitrofurazone and sulfathiazole drugs can lead to systemic side effects. Hence, it is concluded that linarin could be a potent wound healing compound against GSK3 $\beta$.

Keywords: Wound-healing, Glycogen synthase kinase 3 beta, Molecular dynamics, Docking, e-pharmacophore.

(c) 2018 The Authors. Published by Innovare Academic Sciences Pvt Ltd. This is an open access article under the CC BY license (http://creativecommons. org/licenses/by/4. 0/) DOI: http://dx.doi.org/10.22159/ajpcr.2018.v11i2.22962

\section{INTRODUCTION}

A wound is defined as the disruption of the normal anatomical structure and function of a tissue [1]. Healing is the complex and dynamic process that results in the restoration of anatomical continuity and function which consists of three phases: Inflammation, proliferation, and remodeling $[2,3]$. Chronic wounds affect a large number of patients and reduce their quality of life [4]. Medicinal plants have a significant role in the process of wound healing [5]. A large number of plants are used in India for the treatment of cuts, wounds, and burns [6]. Wnt signaling pathway appears to be involved in differentiation processes by controlling the polarity of cell division, cell growth, and cell fate. It is clear that the genes encoding for Wnts and other components of the pathway are expressed during regeneration of the skin [7]. Wnt signaling pathway is divided into two subclasses: The canonical pathway and the non-canonical pathway [8]. Glycogen synthase kinase 3 is a serine/threonine protein kinase that mediates the addition of phosphate molecules on to serine and threonine amino acid residues [9]. Glycogen synthase kinase 3 beta (GSK3 $\beta$ ) is involved in energy metabolism, cell development, and body pattern formation. GSK3 $\beta$ inhibition promotes wound healing through $\beta$-catenindependent Wnt signaling pathway. Several GSK3 $\beta$ inhibitors have been described, and most of the observed effects are in vitro and cellular studies. Among these, inhibitors are paullones, indirubins, 3-anilino-4aryl maleimides, thiadiazolidinones, and other small molecules. Earlier studies reported that in silico findings hypothesized that oleanolic acid may exert the healing activity by inhibiting GSK3 $\beta$ through b-catenindependent Wnt signaling pathway [10]. Similarly, other studies also reported that docking of GSK3 $\beta$ with entagenic acid revealed minimum binding and docking energy than the reference drug nitrofurazone, and thus, it is considered as a good inhibitor of GSK3 $\beta$ [11]. In another study, lupeol was proved to be one of the potent wound healing compounds that show to elicit the cutaneous wound healing better than the reference drug nitrofurazone [12]. Comparative molecular docking of nitrofurazone, sulfathiazole (standard drugs) and entagenic acid, triterpenoid lupeol, and oleanolic acid (phytocompounds) with GSK3 $\beta$ was performed. After docking, the compound showing good interaction was selected for e-pharmacophore modeling and simulation studies [13]. The dataset of 63 compounds was selected based on the literature studies of natural compounds. These wound healing activities of these compounds have been proved through in vitro and animal studies [14]. The present study focuses on in silico inhibition of GSK3 $\beta$ using these natural compounds to find a compound(s) with similar features that can act against GSK3 $\beta$. The structural changes and dynamic behavior of the protein and complex were analyzed by molecular dynamics (MD) studies at $50 \mathrm{~ns}$.

\section{METHODS}

\section{Protein preparation}

The structure of GSK3 $\beta$ (PDB ID: 1Q5K) was downloaded from the protein data bank [7]. Protein preparation wizard tool of Maestro was used for protein preparation. Water molecules which do not participate in interactions were removed, the bond order was assigned, and hydrogen atoms were added. Optimization and minimization of the protein were carried out by applying the OPLS-2005 force field [15].

\section{Ligand preparation}

The structures of the standard drugs (nitrofurazone and sulfathiazole) and phytocompounds (triterpenoid lupeol, entagenic acid, and oleanolic acid) were obtained from the PubChem database [12]. All the ligands were optimized using OPLS_2005 force field, and conformations were generated using Epik in the range of $\mathrm{pH} 7.0 \pm 2.0[15,16]$. The structures were desalted and tautomerized. Thirty-two stereochemical structures were generated per ligand for computation using Ligprep module of Maestro. 
Grid generation

Different ligands which bind within the predicted active site of the receptor grid were generated using Glide receptor grid generation option [17]. Active sites of the protein (Ile62, Gly63, Asn64, Gly65, Ser66, Phe67, Gly68, Val69, Val70, Lys85, Asp181, and Tyr216) were predicted from the CASTp server [18]. The grid was generated keeping the default parameters of van der Waals scaling factor as 1.00 and charge cutoff as 0.25 . Centroid of the selected residues option was selected. The cubic box was set to $10 \AA \times 10 \AA \times 10 \AA$ for docking experiments.

\section{Molecular docking and e-pharmacophore generation}

The two standard drugs and three phytocompounds were docked in the active site of GSK3 $\beta$ using Glide extra precision (XP). All low-energy conformations were determined by Glide XP mode for finding the best conformer with optimum binding affinity. From the results of Glide XP docking, the best conformer was used to generate e-pharmacophore [13]. Script option of Schrodinger phase [19] was used to generate the pharmacophore hypotheses from the docked poses using common set of pharmacophore features, namely, hydrogen bond (H-bond) acceptor (A), H-bond donor (D), hydrophobic group $(\mathrm{H})$, negatively charged group $(\mathrm{N})$, positively charged group $(\mathrm{P})$, and aromatic ring (R). Developed pharmacophore sites were used to filter the leads library with similar pharmacophore groups, and these were given for further XP ligand docking.

\begin{abstract}
Dataset Several phytocompounds were isolated from plants proved to improve wound healing. Sixty-three compounds were selected based on the literature of natural compounds. These compounds wound healing effects have already been proven through in vitro and animal studies, namely, taspine, mesaconitine, hypaconitine, songorine, napelline, 12-epinepheline $\mathrm{N}$-oxide, aucubin, cycloastragenol, catechin, epicatechin-3-0-gallate, quercetin-3-0-methyl ether, anthocyanins, silybin, silychristine, silydianine,1,2,3,4,6-Penta-0-galloyl-beta-Dglucose, embelin, framycetin, proanthycyanidin, resveratrol, epi- $\alpha-$ bisabolol, $\alpha$-bisabolol, $\alpha$-terpineol, asiaticoside, deoxyelephantopin, $\beta$-sitosterol glucoside, $\beta$-sitosterol, emodin, kaempferol, quercetin, quercetin-3-0-rutinoside, protocatechuic acid, P-hydroxybenzoic acid, P-coumaric acid, ferulic acid, vanillic acid, mannose-6-phosphate, emblicanin A, acemannan, spinasterol, stigmasterol, isorhamnetin, xyloglucan, gentiopicroside, deoxyshikonin, acetylshikonin, curcumin, linarin, luteolin, 6-hydroxyluteolin, echinacoside, verbascoside, asiatic acid, madecassic acid, madacassoside, fukinolic acid, cimicifugic acid, madecassol, hypericin, hyperin, vincamine, vinpocetine, and crocetin $[14,20-30]$.
\end{abstract}

\section{E-pharmacophore-based screening}

Developed e-pharmacophore was used as a query for the screening of 63 compounds. Compounds were ranked based on the fitness score above 1.5 [31], and those above this threshold were docked into the active site of the GSK3 $\beta$.

Absorption, distribution, metabolism, and excretion (ADME) screening

The Qikprop program was used to obtain the ADME properties of the compounds [32]. Qikprop predicts the acceptability of the compounds based on Lipinski's rule of five [33]. The final binding pose with the top glide score was selected for further optimization through MD simulations.
MD

MD simulations were performed to evaluate the stability. Simulations were carried out using Desmond v3.0 in Schrodinger package. Single point charge (SPC) system was used to mimic the physiological behavior of the molecules. Minimization was done using OPLS 2005 force field. Boundary condition was set as orthorhombic, and the system was neutralized by adding counter ions. The system was minimized with maximum 2000 iterations with a convergence threshold of $1.00 \mathrm{kcal} /$ mol [34]. The MD simulation was carried out at 50 ns. A constant temperature of $300 \mathrm{~K}$ was maintained throughout the simulation. Rootmean-square deviation (RMSD), root-mean-square fluctuation (RMSF), and $\mathrm{H}$-bonds interactions were analyzed.

\section{RESULTS}

Molecular docking interaction of GSK-3 $\beta$ with standard drugs and phytocompounds triterpenoid lupeol, oleanolic acid, and entagenic acid

Standard drugs (nitrofurazone and sulfathiazole) and phytocompounds (triterpenoid lupeol, oleanolic acid, and entagenic acid) were docked with GSK3 $\beta$ (Fig. 1). The Glide XP docking of GSK3 $\beta$ with entagenic acid revealed minimum glide score and glide energy when compared with the other ligands (Table 1). XP docking interaction of entagenic acid with GSK3 $\beta$ showed a glide score of $-4.31 \mathrm{kcal} / \mathrm{mol}$. Four hydrogen bonding interactions were seen with the amino acid residues Asp200, Asn186, Asp181, and Arg144 (Fig. 2).

Dynamic analysis of RMSD value for Apo (GSK3 $\beta$ ), GSK3 $\beta$ nitrofurazone complex, and entagenic acid complex

The dynamic behavior of apo (GSK3 $\beta$ ), GSK3 $\beta$-nitrofurazone, and GSK3 $\beta$-entagenic acid complexes was analyzed using RMSD. RMSD is the measure of the average distance between the atoms of superimposed proteins (Fig. 3). For the backbone atom of apo (GSK3 $\beta$ ) protein, RMSD value increased from 0.82 to $1.00 \AA \AA$. From 38 picoseconds (ps) to $50 \mathrm{~ns}$, the RMSD kept fluctuating between 2.0 and $2.7 \AA$. The overall interacting distance was fluctuating between 0.82 and $2.7 \AA$. The average interacting distance was observed as $2.11 \AA$. For GSK3 $\beta$-nitrofurazone complex, the RMSD value increased from 0.73 to $1.08 \AA$. It remained relatively stable from 43 ps to $9465 \mathrm{ps}$, and the fluctuation range was observed to be 0.95-1.92 Å. From 9466 to $50 \mathrm{~ns}$, the deviation range was from 1.68 to $2.67 \AA$. The overall interacting distance was fluctuating between 0.73 and $2.67 \AA$. The average interacting distance was observed as $2.02 \AA$. For GSK3 $\beta$-entagenic acid complex, RMSD value increased from $0.68 \mathrm{~A}$ to $1.02 \AA$. From 24 ps onward, the RMSD kept fluctuating between 0.84 and $2.45 \AA$. The overall interacting distance was fluctuating between 0.68 and $2.45 \AA$. The average interacting distance was observed to be $1.98 \AA$. The result indicates that GSK3 $\beta$-entagenic complex showed lower RMSD deviation throughout the simulation when compared with the apo (GSK3 $\beta$ ) and GSK3 $\beta$-nitrofurazone complex.

Dynamic analysis of RMSF value for Apo (GSK3 $\beta$ ), GSK3 $\beta$ nitrofurazone complex, and GSK3 $\beta$-entagenic acid complex RMSF was used as a measure of conformational variance. RMSF values of the backbone atoms of apo (GSK3 $\beta$ ) decreased from 3.96 to 0.51 A initially (Fig. 4). The value remained low until residue 122 , where it increased to $4.86 \AA$. From 123 to 379 residues, the deviation range was from 0.42 to $3.43 \AA$. Highest RMSF value of $8.79 \AA$ was obtained at the final residue, 384 . For GSK3 $\beta$-nitrofurazone complex, the fluctuation mostly decreased initially, with the range being from

Table 1: Glide score, glide energy, and glide emodel of GSK3 $\beta$ with standard drugs and phytocompounds

\begin{tabular}{llllll}
\hline S. No. & Inhibitors & Glide score (kcal/mol) & Glide energy (kcal/mol) & Glide emodel (kcal/mol) & H-bond \\
\hline 1 & Entagenic acid & -4.31 & -46.11 & -46.55 & -1.62 \\
2 & Oleanolic acid & -4.27 & -39.84 & -56.37 & -2.46 \\
3 & Nitrofurazone & -3.32 & -30.60 & -39.14 & -1.10 \\
4 & Sulfathiazole & -3.31 & -26.21 & -35.40 & -0.70 \\
5 & Triterpenoid lupeol & -3.27 & -35.43 & -42.88 & -0.77 \\
\hline
\end{tabular}

GSK3ß: Glycogen synthase kinase 3 beta 


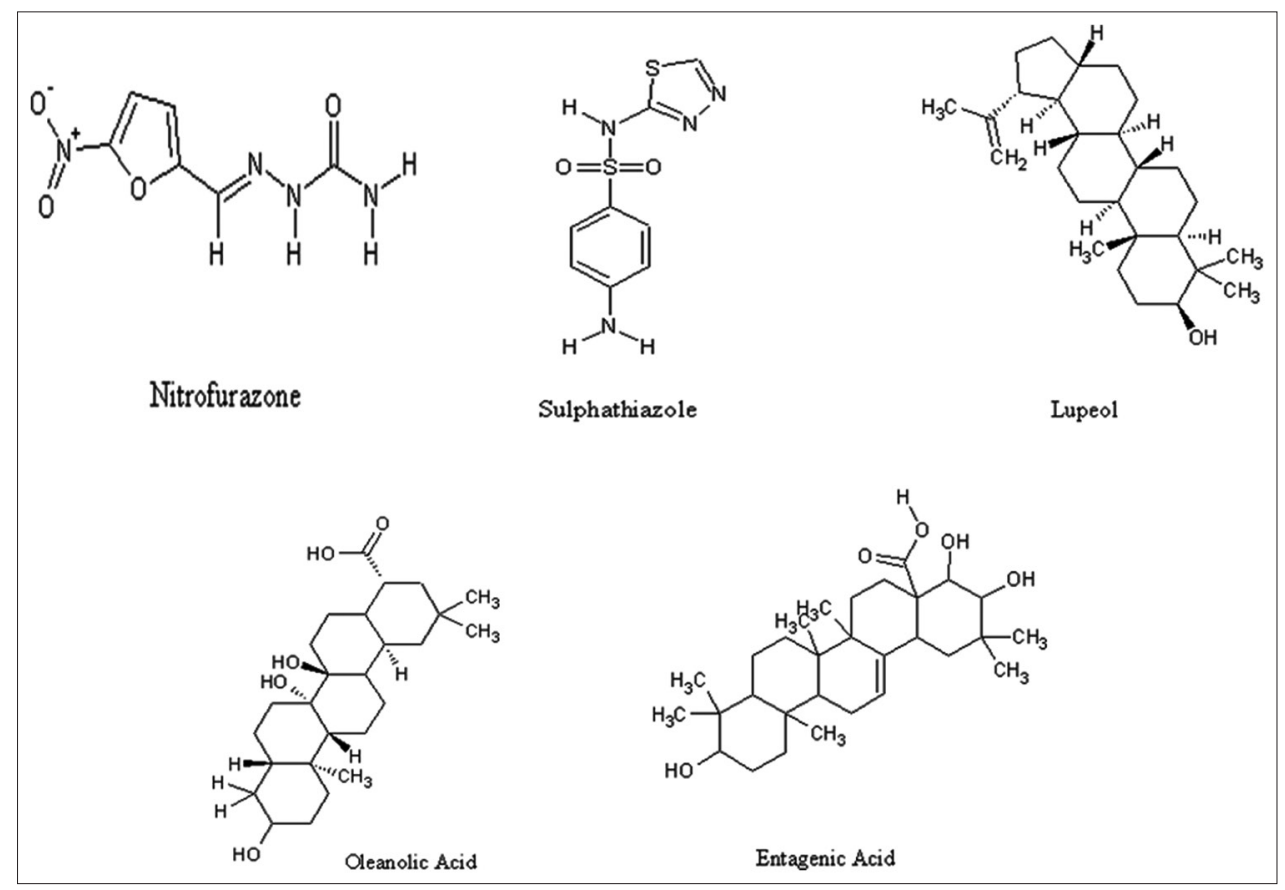

Fig. 1: Structures of the drugs and phytocompounds

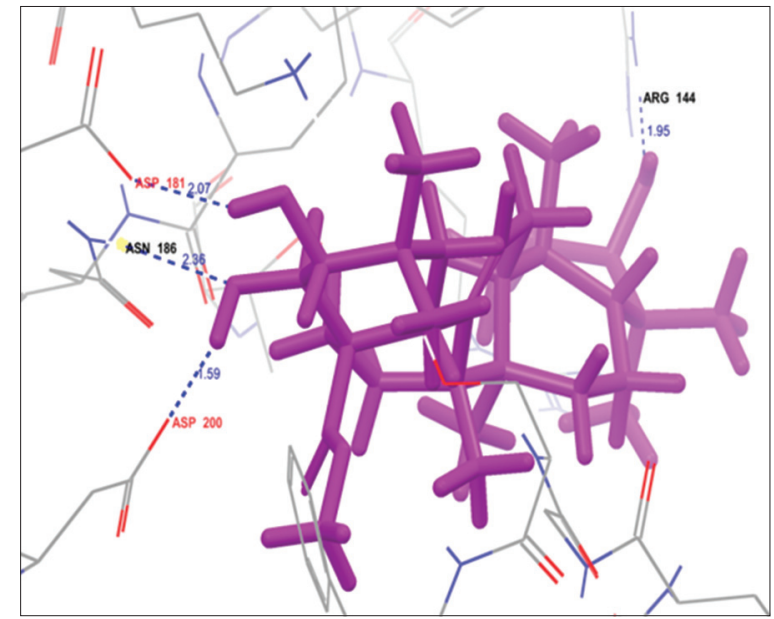

Fig. 2: Interaction of glycogen synthase kinase 3 beta with entagenic acid showing hydrogen bond

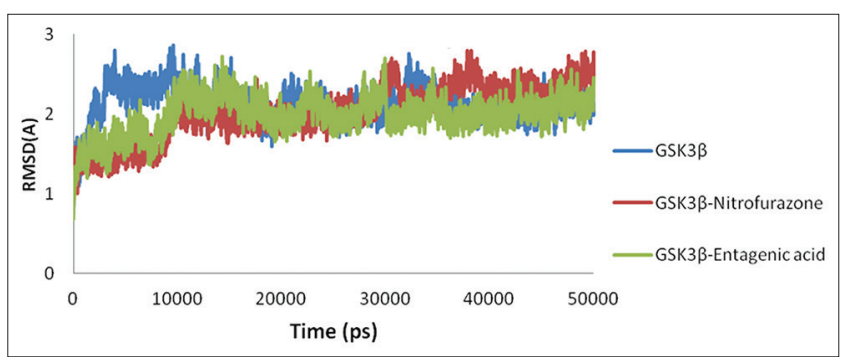

Fig. 3: Root-mean-square deviation of backbone atoms of apo (glycogen synthase kinase 3 beta [GSK3 $\beta$ ]), GSK3 $\beta$-nitrofurazone complex, and GSK3 $\beta$-entagenic acid complex during $50 \mathrm{~ns}$

3.61 to $0.63 \AA$. The value then increased to $4.18 \AA$ at residue 122 . Most of the residues were flexible and usually lie below $4.0 \AA$, except the residues 286, 381, 382, 383, and 384. For GSK3 $\beta$-entagenic complex, the value increased from 0.41 to $4.85 \AA$. The majority

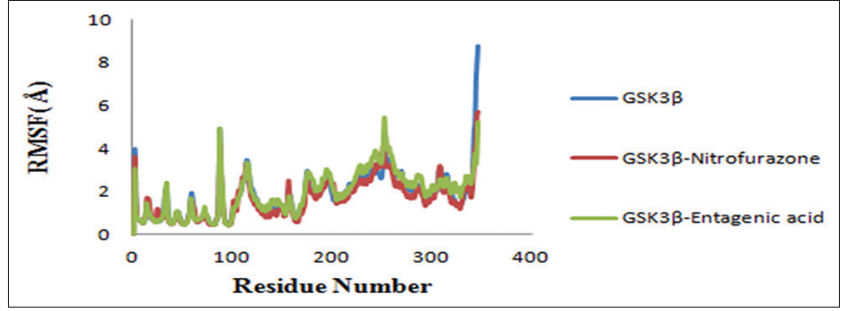

Fig. 4: Root-mean-square fluctuations of the amino acid of apo glycogen synthase kinase 3 beta (GSK3 $\beta$ ), GSK3 $\beta$-nitrofurazone complex, and GSK3 $\beta$-Entagenic acid complex at 50 ns

of the residues were flexible, and their RMSF was observed below 4.0 Å. Some residues such as $286,290,291,292$, and 384 showed fluctuation difference of $4.29,5.44,4.72,4.45$, and $5.82 \AA$. The overall range was $0.42-8.79 \AA$ for apo (GSK3 $\beta$ ), $0.40-5.74 \AA$ for GSK3 $\beta$ nitrofurazone complex, and $0.40-5.22 \AA$ for the GSK3 $\beta$-entagenic acid complex. When compared to apo (GSK3 $\beta$ ) and GSK3 $\beta$-nitrofurazone complex, the GSK3 $\beta$-entagenic acid complex showed low fluctuations for amino acid residues Arg141 and Arg144.

Analysis of H-bond interactions of GSK3 $\beta$-nitrofurazone complex and GSK3 $\beta$-entagenic acid complex

The stability of protein-ligand was analyzed using H-bond interaction. Throughout the simulation, GSK3 $\beta$-nitrofurazone complex showed many $\mathrm{H}$-bonds in the range of $0-2$. In a few trajectories, 3 and $4 \mathrm{H}$-bond interactions were observed. For GSK3 $\beta$-entagenic acid complex, $\mathrm{H}$-bond interaction was observed to be mostly in the range of 2-3 throughout the simulation. Few trajectories even showed 4 and 5 bonds. This $\mathrm{H}$-bond interaction analysis clearly indicates that this ligand maintains stability during the MD studies (Fig. 5). The H-bond interaction of GSK3 $\beta$-entagenic acid complex was observed at different ns. Arg141, Lys183, Asn186, and Asp200 showed H-bond interaction at 0 ns. No interaction was observed at $10 \mathrm{~ns}$. The 20 ns showed the H-bond interaction with Asn186. No interaction was observed at 30 and 40 ns. Gly185 showed interaction at 50 ns. H-bond interactions in Gly185 and Asn186 are seen to be stable in most of the trajectories, indicating that these bonds are most stable. Some trajectories showed Lys183 and Asp 200 forming $\mathrm{H}$-bonds with water bridges. 
E-pharmacophore hypotheses generation and screening

E-pharmacophore was developed based on the docked complex of entagenic acid. The Glide XP results were used for building the e-pharmacophore from the Maestro Scripts menu option with default excluded volume option 0.5. Five pharmacophore features were predicted. The final hypothesis consists of three H-bond acceptor (A) and two H-bond donor (D), and their distances have been shown in Fig. 6 and Table 2. The pharmacophore model of entagenic acid created was screened with a dataset of 63 compounds to find similar compounds. 18 compounds with Fitness Score above 1.5 were selected from a total of 63 compounds (Table 3 ).

\section{XP docking interaction of GSK3 $\beta$ with top 18 hits}

Eighteen compounds with fitness score above 1.5 were docked with GSK3 $\beta$ using Glide XP docking. Glide score, Glide energy, and H-bond interactions were used to predict the best conformation. The compounds with more than $-4.0 \mathrm{kcal} / \mathrm{mol}$ glide score are given in Table 4 .

\section{ADME properties}

The 18 compounds obtained from docking were screened for ADME properties. It has been observed that few compounds showed the poor drug-likeness score. Compounds with significant ADME properties have been reported in Table 5.

\section{Docking interactions of the selected phytocompounds}

Of all the interactions of phytocompounds with GSK3 3 , docked orientation of the fukinolic acid showed the glide score $-10.99 \mathrm{kcal} /$ mol, glide energy $-49.88 \mathrm{kcal} / \mathrm{mol}$, glide emodel $-58.94 \mathrm{kcal} / \mathrm{mol}$, and four H-bond interactions. Cimicifugic acid showed glide score $-8.28 \mathrm{kcal} / \mathrm{mol}$, glide energy $-42.09 \mathrm{kcal} / \mathrm{mol}$, and glide emodel -49.96 $\mathrm{kcal} / \mathrm{mol}$ at the active site of GSK3 $\beta$. Four H-bond interactions were observed. Other noteworthy compound linarin showed glide score $-7.53 \mathrm{kcal} / \mathrm{mol}$, glide energy $-60.48 \mathrm{kcal} / \mathrm{mol}$, glide emodel -72.69 $\mathrm{kcal} / \mathrm{mol}$, and five H-bond interactions (Table 6 and Fig. 7).

Table 2: Pharmacophore site features for entagenic acid using phase

\begin{tabular}{lll}
\hline Rank & Features & Score \\
\hline 1 & D8 & -0.8 \\
2 & D7 & -0.79 \\
3 & A2 & -0.5 \\
4 & A1 & -0.33 \\
5 & A3 & -0.06 \\
\hline
\end{tabular}

Table 3: Fitness score for the top 18 hits screened through e-pharmacophore hypothesis

\begin{tabular}{ll}
\hline Compound name & Fitness score \\
\hline Madaecassol & 2.03 \\
Isorhamnetin & 1.53 \\
Cimicifugic acid & 1.68 \\
Fukinolic acid & 1.76 \\
Linarin & 1.50 \\
Verbascoside & 1.82 \\
Echinacoside & 1.87 \\
Hyperin & 1.75 \\
Quercetin-3-o-rutinoside & 1.78 \\
Cynaroside & 1.73 \\
Madacassoside & 2.15 \\
Isoorientin & 1.76 \\
Asiaticoside & 2.14 \\
Epicatechin-3-o-gallate & 1.57 \\
Gentiopicroside & 1.85 \\
1,2,3,4,6-Penta-o-galloyl-beta-D-glucose & 1.72 \\
Framycetin & 1.53 \\
P-hydroxybenzoic acid & 2.09 \\
\hline
\end{tabular}

Dynamics analysis of RMSD value for GSK3 $\beta$ with selected phytocompounds

For the backbone atom of GSK3 $\beta$-linarin complex, RMSD value increased from 0.67 to $1.06 \AA$ (Fig. 8). From 67 ps to the end of the simulation, the interacting distance kept fluctuating between 0.98 and $2.46 \AA$. The average interacting distance was observed as $1.97 \AA$. The RMSD backbone atom of GSK3 $\beta$-fukinolic acid complex showed an increase from 0.76 to $1.15 \AA$. From 57 to the end of the simulation, the interacting distance kept fluctuating between 1.07 and $3.05 \AA$. The average interacting distance was observed as $2.35 \AA$. The RMSD backbone atom of GSK3 $\beta$-cimicifugic acid complex showed an increase from 0.66 to $1.06 \AA$. From 43 to the end of the simulation, the interacting distance kept fluctuating between 0.9 and $2.61 \AA$. The average interacting distance was observed as $1.97 \AA$. The result indicates that GSK3 $\beta$-linarin complex showed lower RMSD deviation throughout the simulation when compared to other ligands.

Dynamic analysis of RMSF value for GSK3 $\beta$ with selected phytocompounds

For GSK3 $\beta$-entagenic acid complex, RMSF value decreased from 3.03 to $0.66 \AA$. The range was observed to be $0.40-5.22 \AA$ throughout the simulation (Fig. 9). For GSK3 $\beta$-linarin complex, RMSF decreased from 4.8 to $0.52 \AA$, and overall range was from 0.45 to $6.11 \AA$. Most of the residues were flexible and lie below $4.0 \AA$. For GSK3 $\beta$-fukinolic acid complex, the RMSF value decreased from 5.07 to $0.69 \AA$, and the range was observed between 0.46 and $6.87 \AA$. The majority of the residues were flexible, and their RMSF was well below $4 \AA$. Some residues such as $35,122,382,383$ and 384 showed fluctuation difference of 5.07, $5.21,4.88,6.71$, and 6.87. For GSK3 $\beta$-cimicifugic acid complex, RMSF value decreased from 3.33 to $0.72 \AA$, and the range was between 0.46 and $6.39 \AA$. Most residues were flexible and below $4 \AA$, except the residues $122,277-279,286-99$, and 384 . The result indicates that linarin complex showed low RMSF deviation throughout the simulation when compared with other ligands.

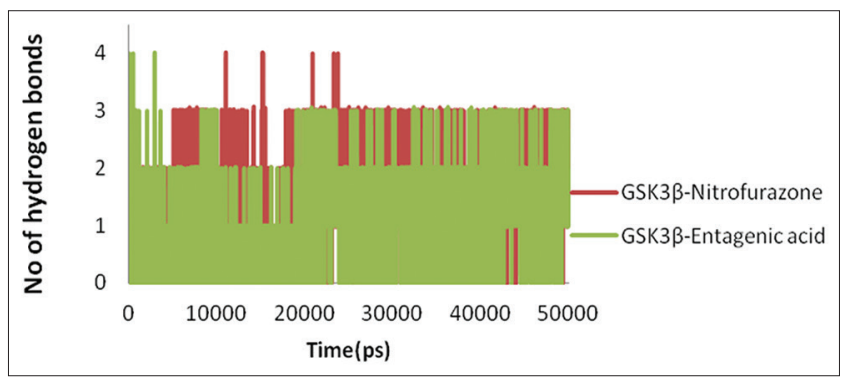

Fig. 5: Number of hydrogen bonds for glycogen synthase kinase 3 beta (GSK3 $\beta$ )-nitrofurazone complex and GSK3 $\beta$-entagenic acid complex at $50 \mathrm{~ns}$

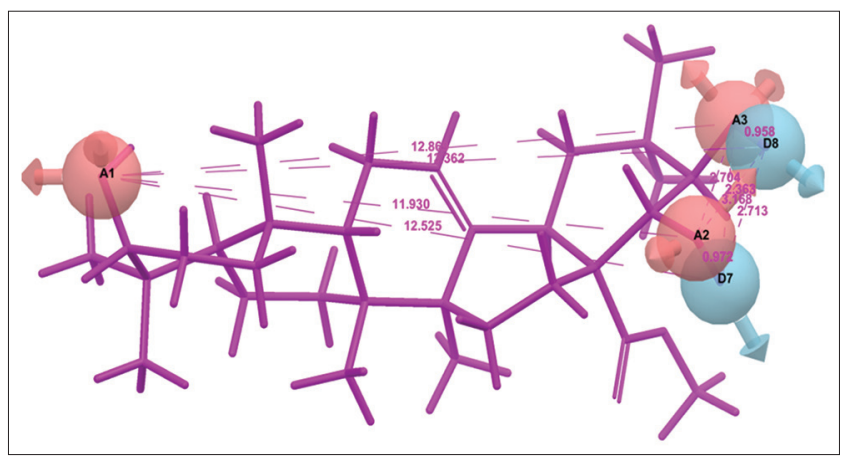

Fig. 6: Pharmacophore hypothesis AAADD (A: Hydrogen acceptor and D: Hydrogen donor) generated for entagenic acid using script-dock post-processing 


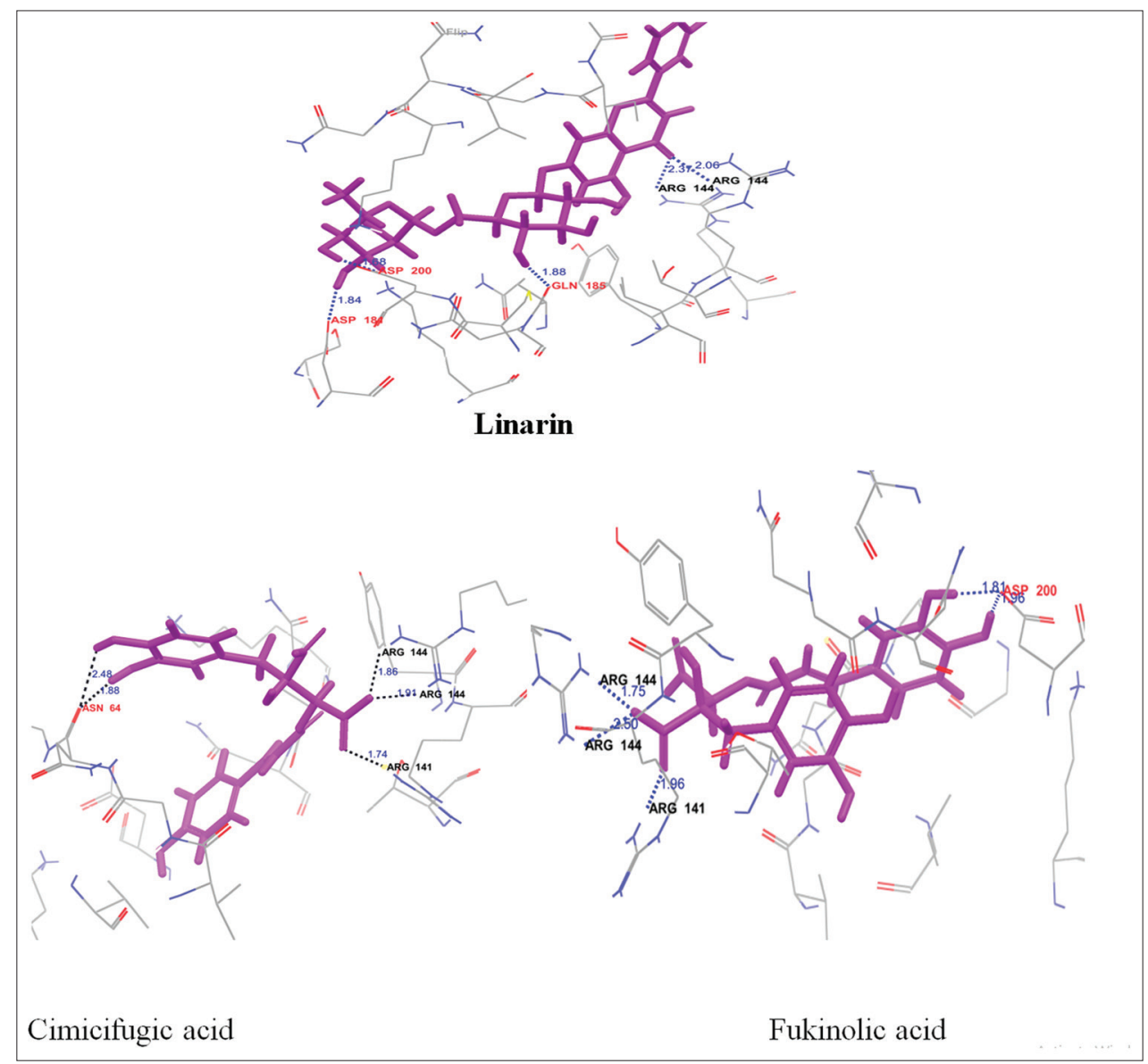

Fig. 7: Interactions of glycogen synthase kinase 3 beta with selected phytocompounds linarin, cimicifugic acid, and fukinolic acid

Table 4: Glide score, glide energy, and glide emodel of GSK3 $\beta$ with top 18 hits

\begin{tabular}{|c|c|c|c|c|}
\hline Compound name & Glide score (kcal/mol) & Glide energy (kcal/mol) & Glide emodel (kcal/mol) & H-bond \\
\hline 1,2,3,4,6-Penta-o-galloyl- $\beta$-D-glucose & -13.34 & -95.38 & -97.34 & -8.80 \\
\hline Fukinolic acid & -10.99 & -49.88 & -58.94 & -4.65 \\
\hline Madaecassol & -10.94 & -67.29 & -98.68 & -6.58 \\
\hline Echinacoside & -10.57 & -69.38 & -99.18 & -5.72 \\
\hline Madacassoside & -10.02 & -58.88 & -46.75 & -6.24 \\
\hline Cynaroside & -9.22 & -51.01 & -60.04 & -5.63 \\
\hline Epicatechin-3-o-gallate & -8.35 & -53.69 & -68.60 & -4.37 \\
\hline Cimicifugic acid & -8.28 & -42.09 & -49.96 & -3.14 \\
\hline Quercetin-3-o-rutinoside & -8.17 & -64.59 & -85.56 & -4.17 \\
\hline Verbascoside & -7.87 & -46.13 & -76.19 & -4.45 \\
\hline Framycetin & -7.54 & -58.49 & -67.79 & -5.77 \\
\hline Linarin & -7.53 & -60.48 & -72.69 & -4.07 \\
\hline Asiaticoside & -7.21 & -55.69 & -57.04 & -3.76 \\
\hline Hyperin & -6.64 & -48.44 & -52.19 & -3.20 \\
\hline Isoorientin & -6.20 & -55.64 & -56.85 & -4.02 \\
\hline Isorhamnetin & -5.83 & -42.39 & -52.89 & -2.66 \\
\hline P-hydroxybenzoic acid & -5.58 & -43.10 & -54.09 & -1.96 \\
\hline Gentiopicroside & -4.89 & -37.72 & -44.89 & -1.43 \\
\hline
\end{tabular}

GSK3ß: Glycogen synthase kinase 3 beta

Table 5: Predicted drug-likeness properties of selected phytocompounds

\begin{tabular}{lllll}
\hline Compound name & Molecular weight & Hydrogen bond donor & Hydrogen-bond acceptor & Lipophilicity \\
\hline Isorhamnetin & 316.27 & 4 & 6.25 & 0.88 \\
Cimicifugic acid & 418.36 & 5 & 8 & 1.61 \\
Gentiopicroside & 356.33 & 4 & 14.9 & -1.53 \\
Fukinolic acid & 434.36 & 6 & 8.75 & 0.95 \\
Cynaroside & 448.38 & 6 & 13 & -1.06 \\
Linarin & 592.55 & 6 & 19.05 & -0.75 \\
\hline
\end{tabular}


Table 6: Glide score, glide energy, glide emodel, and H-bond interactions of GSK3 $\beta$ with selected phytocompounds

\begin{tabular}{lllll}
\hline Name & Glide score $\mathbf{( k c a l / m o l )}$ & Glide energy (kcal/mol) & Glide emodel (kcal/mol) & H-bond interactions \\
\hline Fukinolic acid & -10.99 & -49.88 & -58.94 & Arg144, Asp141, Asp200 \\
Cimicifugic acid & -8.28 & -42.09 & -49.96 & Arg144, Arg141, Asn64 \\
Linarin & -7.53 & -60.48 & -72.69 & Asp181, Asp200, Gln185, Arg144 \\
\hline
\end{tabular}

GSK3ß: Glycogen synthase kinase 3 beta

Analysis of H-bond interactions of GSK3 $\beta$ with selected phytocompounds

GSK3 $\beta$-linarin complex showed H-bond interactions with Asp200, Lys183, and Thr138 at 0 ns, Lys183, Asp200, and Gly185 at 10 ns, Asp200 and Lys183 at 20-30 ns, and there was no interaction observed at 40 ns (Fig. 10). Similarly, H-bonds were observed with residues Thr138, Asp200, and Lys183 at $50 \mathrm{~ns}$. In all of the trajectories, H-bonding with Asp200 and Lys183 was observed. In the range of 0 to $50 \mathrm{~ns}$ in most of the trajectories, 3-6 H-bonds were maintained. For GSK3 $\beta$-cimicifugic acid complex, H-bonds were observed with residues Arg144 and Arg141 at 0 ns, with residues Gly185 and Arg144 at 10 ns, and with Arg144 and Arg 141 at 20 as well as at 30 ns. H-bonding was also observed with Arg144 at 40 and 50ns. Interaction with Arg144 was observed throughout the simulation. Most of the trajectories showed interaction with Arg141. From 0 to $50 \mathrm{~ns}$, it maintains 2-3 H-bonds. Only a few trajectories showed 5 H-bonds. For GSK3 $\beta$-fukinolic acid docking complex, H-bonding was observed with residues Lys183, Asp200, Arg144, and Arg141 at 0 ns, with Asp200 and Arg141 at 10 ns, and with Arg144 at 20-50 ns. Most of the trajectories showed interaction with Arg144. Arg141 was also observed to be interacting in few trajectories. From $1 \mathrm{~ns}$ to the end, 1-2 H-bonds were observed. Few trajectories showed the presence of 4- $5 \mathrm{H}$-bonds. These results indicate that H-Bond interactions of GSK3 $\beta$-linarin complex remained consistent throughout the MD simulation when compared with GSK3 $\beta$ cimicifugic and GSK3 $\beta$-fukinolic complexes.

\section{DISCUSSION}

Wound healing is a complex and dynamic process of restoring cellular structures and tissue layers in damaged tissue as closely as possible to its normal state [11]. The earlier reports indicated that Wnts are necessary for normal skin development [12]. GSK3 $\beta$ inhibition promotes wound healing through $\beta$-catenin-dependent Wnt signaling pathway. Several GSK3 $\beta$ inhibitors have been described, and most of the observed effects are in vitro and cellular studies. Docking of phytocompounds, oleanolic acid from the methanolic extract of the bark of Grewia tiliaefolia, entagenic acid from seed kernel of Entada pursaetha, lupeol from petroleum ether extract of leaves of Celastrus paniculatus, and the standard drugs nitrofurazone and sulfathiazole with GSK3 $\beta$ has been reported [10-12]. Nitrofurazone and nitrofuran family drugs showed side effects leading to increased cell killing in some individuals [35]. Due to this and also an increased observation of sensitization in patients treated with sulfathiazole, there is a necessity for finding a new drug [36]. Phytocompounds from plants are used to treat various diseases and showed fewer side effects [37]. In this study, the wound healing phytocompound entagenic acid, lupeol, and oleanolic acid are docked with the receptor GSK3 $\beta$ and compared with standard drugs nitrofurazone and sulfathiazole to assess the comparative wound healing potency. The purpose of the study was to compare wound healing compounds and standard drugs and analyze the docking mode of these compounds on the target site. It was observed that the XP interaction of entagenic acid with GSK3 $\beta$, the minimum glide score of $-4.31 \mathrm{kcal} / \mathrm{mol}$ when compared to other compounds and standard drugs. Entagenic acid showed 4 hydrogen bonding interactions with residues Asp200, Asn186, Asp181, and Arg144. Further dynamic results confirmed that this compound remained stable in most of the trajectory. This complex was used to predict the e-pharmacophore hypothesis. The generated hypothesis model consists of three H-bond acceptor (A) and two H-bond donor (D). Sixty-three compounds were selected based on the literature of natural compounds, and the wound

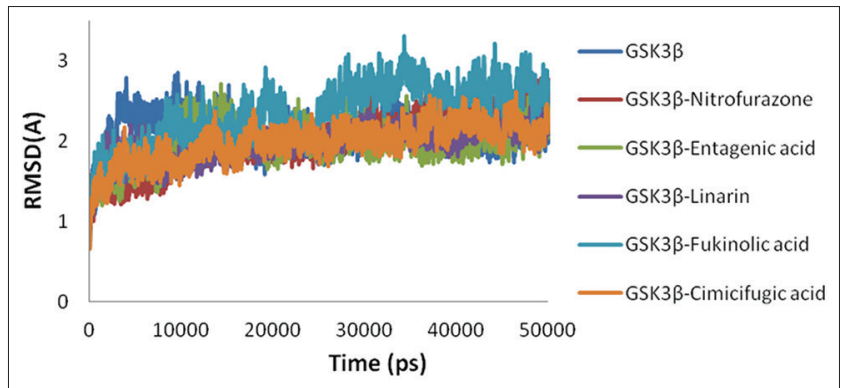

Fig. 8: Root-mean-square deviations of backbone atoms of glycogen synthase kinase 3 beta (GSK3 $\beta$ )-entagenic acid complex

compared against GSK3 $\beta$-linarin, GSK3 $\beta$-fukinolic acid, and GSK3 $\beta$-cimicifugic acid complexes at $50 \mathrm{~ns}$

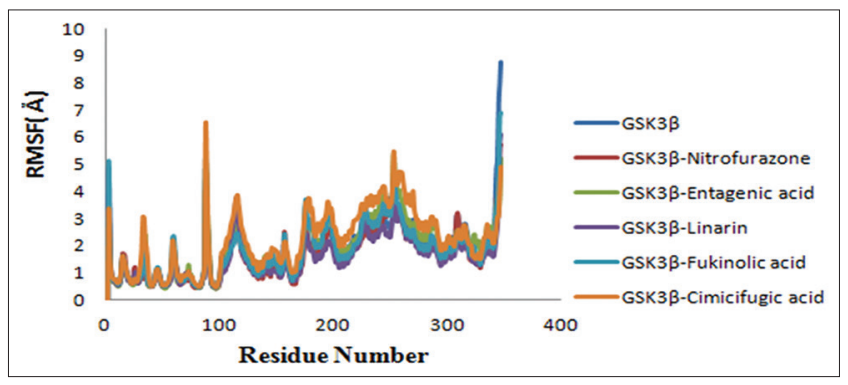

Fig. 9: Root-mean-square fluctuations for a specific atom of glycogen synthase kinase 3 beta-entagenic acid complex with linarin, fukinolic acid, and cimicifugic acid complexes at $50 \mathrm{~ns}$

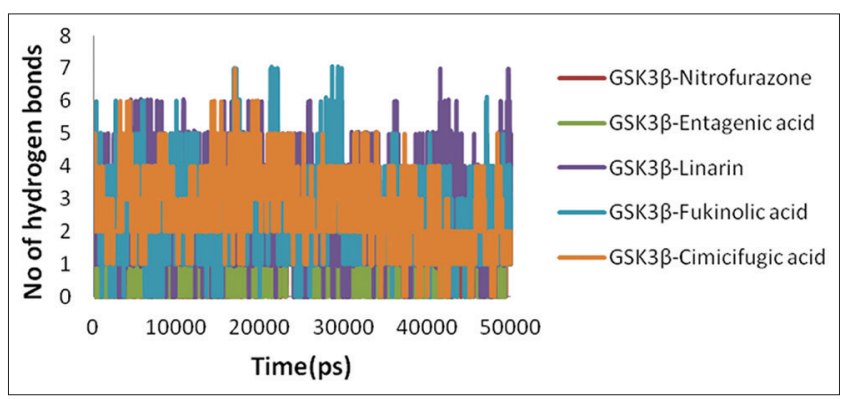

Fig. 10: H-bond interactions for a specific atom of glycogen synthase kinase 3 beta (GSK3 $\beta$ )-linarin, GSK3 $\beta$-fukinolic acid, and GSK3 $\beta$-cimicifugic acid complexes

healing effects have already been proven through in vitro and animal studies, but in silico studies against our target GSK3 $\beta$ have not been reported. These phytocompounds were screened against the generated hypothesis AAADD of entagenic acid. The compounds with fitness score of more than 1.5 were further subjected to XP docking [38]. 18 compounds had better glide score when compared with entagenic acid, only a few compounds had satisfactory ADME properties. The docking results of GSK3 $\beta$-linarin complex (5317025), GSK3 $\beta$-fukinolic acid complex (6441059), and GSK3 $\beta$-cimicifugic acid complex (6450178) were compared based on the various parameters such as the glide score, glide energy, glide emodel, and H-bond interactions. Glide XP interaction of GSK3 $\beta$-fukinolic acid, cimicifugic acid, and linarin complexes showed 
the glide score of $-10.99,-8.28$, and $-7.52 \mathrm{kcal} / \mathrm{mol}$, glide energy value of $-49.88,-42.09$, and $-60.48 \mathrm{kcal} / \mathrm{mol}$, and glide emodel of -58.94 , -49.96 , and $-72.69 \mathrm{kcal} / \mathrm{mol}$, respectively. $\mathrm{H}$-bond interactions of GSK3 $\beta$-linarin, fukinolic acid, and cimicifugic acid complexes were compared. All the three complexes showed more than three H-bonds. Hence, these three compounds were taken for dynamics simulation. Even though the glide score of linarin was low when compared to cimicifugic acid and fukinolic acid, linarin was chosen for further investigation since $\mathrm{H}$-bond interactions were given utmost priority for the study. If the H-bond interaction is more, the binding affinity of the ligand is higher [39]. In GSK3 $\beta$-linarin complex, five $\mathrm{H}$-bonds were seen. The distances of the $\mathrm{H}$-bonds were $<3$ which indicated the presence of favorable interactions. The results also support that the glide energy of linarin was found to be $-60.48 \mathrm{kcal} / \mathrm{mol}$ indicating excellent binding affinity. The low glide emodel value $-72.69 \mathrm{kcal} / \mathrm{mol}$ of linarin indicates the best binding affinity between protein and ligand. Furthermore, MD results of GSK3 $\beta$-linarin complex showed low RMSD deviation throughout the simulation when compared to other ligands. Minimum RMSF deviations were seen in residues Arg141, Arg144, Asp181, Gln185, Asn186, and Asp200, indicating stable conformation. It is clear from the H-bond graph that linarin had the maximum number of $\mathrm{H}$-bonds. Earlier works reported that linarin was identified in Buddleja globosa, Buddleia cordata, Buddleia Perfoliata, and Buddleia scordioides [21,23]. Linarin is a flavonoid which possesses anti-inflammatory, analgesic, antipyretic, anti-oxidative, diaphoretic, hypotensive, anti-stress/ anxiety, antithrombotic, and wound healing properties $[23,24,40]$. It could potentially activate macrophages by producing cytokines [41]. Collectively, RMSD, RMSF, and H-bond of dynamics study determined that the GSK3 $\beta$-linarin complex was stable at 50 ns when compared to GSK3 $\beta$-fukinolic acid and GSK3 $\beta$-cimicifugic acid complexes. The present study suggests that of the 63 natural compounds proven to possess wound healing properties, linarin could potentially be the best inhibitor for GSK3 $\beta$.

\section{CONCLUSIONS}

Nitrofurazone and sulfathiazole drugs can lead to side effects; there is a need for the development of new drugs for wound healing. Therefore, we can conclude that our study addressed on comparison among the three experimentally proven phytocompounds (entagenic acid, lupeol, and oleanolic acid) and standard drugs (nitrofurazone and sulfathiazole), an entagenic acid found to better in XP docking with GSK3 $\beta$. Further, dynamics results confirmed this compound remained stable at 50 ns. E-pharmacophore hypothesis generated based on the GSK3 $\beta$-entagenic acid complex was further used to screen active compounds from a dataset of 63 compounds. The shortlisted three compounds showed better interactions with GSK3 $\beta$ with high fitness score, glide score, and glide energy, and ADME properties were further taken for dynamics simulation at 50 ns. The GSK3 $\beta$-linarin showed a maximum number of $\mathrm{H}$-bond interaction, low RMSD deviation, and minimum RMSF fluctuation when compared with entagenic acid. Hence, it is concluded that linarin could be a potent inhibitor of GSK3 $\beta$ and could be an effective wound healer.

\section{ACKNOWLEDGEMENT}

The authors would like to thank SRM University for their constant support.

\section{CONFLICTS OF INTERESTS}

The authors have none to declare.

\section{AUTHORS CONTRIBUTION}

JBS and WH designed and performed the experiment. JBS and WH wrote the manuscript.

\section{REFERENCES}

1. Diegelmann RF, Evans MC. Wound healing: An overview of acute, fibrotic and delayed healing. Front Biosci 2004;9:283-9.

2. Lazarus GS, Cooper DM, Knighton DR, Margolis DJ, Pecoraro RE, Rodeheaver $\mathrm{G}$, et al. Definitions and guidelines for assessment of wounds and evaluation of healing. Arch Dermatol 1994;130:489-93.

3. Kumarasamyraja D, Jeganathan NS, Manavalan R. A review on medicinal plants with potential wound healing activity. Int J Pharm Pharm Sci 2012;2:105-11.

4. Hayouni EA, Miled K, Boubaker S, Bellasfar Z, Abedrabba M, Iwaski $\mathrm{H}$, et al. Hydroalcoholic extract based-ointment from Punica granatum L. Peels with enhanced in vivo healing potential on dermal wounds. Phytomedicine 2011;18:976-84.

5. Thakur R, Jain N, Pathak R, Sandhu SS. Practices in wound healing studies of plants. Evid Based Complement Alternat Med 2011;2011:438056.

6. Kumari P, Yadav P, Verma PR, Kumar S, Arya A. A review on wound healing properties of Indian medicinal plants. Indian J Fundam Appl Life Sci 2013;3:220-32.

7. Paramesha M, Ramesh CK, Krishna V, Swamy HM, Rao SA, Hoskerri J. Effect of dehydroabietylamine in angiogenesis and GSK3- $\beta$ inhibition during wound healing activity in rats. Med Chem Res 2015;24:295-303.

8. Zhang DL, Gu LJ, Liu L, Wang CY, Sun BS, Li Z, et al. Effect of wnt signaling pathway on wound healing. Biochem Biophys Res Commun 2009;378:149-51.

9. Gokhale KM, Tilak BP. Role of glycogen synthase kinase (GSK-3) in Type-2 diabetes and GSK-3 inhibitors as potential anti-diabetics. Int J Pharm Phytopharm Res 2017;3:196-9.

10. Ahamed KB, Gowdru HB, Rajashekarappa S, Malleshappa KS, Krishna V. Molecular docking of glycogen synthase kinase 3- $\beta$ inhibitor oleanolic acid and its wound-healing activity in rats. Med Chem Res 2013;22:156-64.

11. Vidya SM, Krishna V, Manjunatha BK, Bharath BR, Rajesh KP, Manjunatha $\mathrm{H}$, et al. Wound healing phytoconstituents from seed kernel of Entada pursaetha DC. and their molecular docking studies with glycogen synthase kinase 3- $\beta$. Med Chem Res 2012;21:3195-203.

12. Harish BG, Krishna V, Santosh Kumar HS, Khadeer Ahamed BM, Sharath R, Kumara Swamy HM, et al. Wound healing activity and docking of glycogen-synthase-kinase-3-beta-protein with isolated triterpenoid lupeol in rats. Phytomedicine 2008;15:763-7.

13. Nagamani S, Kesavan C, Muthusamy K. E-pharmacophore mapping and docking studies on vitamin $\mathrm{D}$ receptor (VDR). Bioinformation 2012;8:705-10.

14. Tsala DE, Amadou D, Habtemariam S. Natural wound healing and bioactive natural products. Phytopharmacology 2013;4:532-60.

15. Chen IJ, Foloppe N. Drug-like bioactive structures and conformational coverage with the ligPrep/ConfGen suite: Comparison to programs MOE and catalyst. J Chem Inf Model 2010;50:822-39.

16. Shelley JC, Cholleti A, Frye LL, Greenwood JR, Timlin MR, Uchimaya M, et al. Epik: A software program for $\mathrm{pK}(\mathrm{a})$ prediction and protonation state generation for drug-like molecules. J Comput Aided Mol Des 2007;21:681-91.

17. Friesner RA, Murphy RB, Repasky MP, Frye LL, Greenwood JR, Halgren TA, et al. Extra precision glide: Docking and scoring incorporating a model of hydrophobic enclosure for protein-ligand complexes. J Med Chem 2006;49:6177-96.

18. Rayalu DJ, Selvaraj C, Singh SK, Ganeshan R, Kumar NU, Seshapani P, et al. Homology modeling, active site prediction, and targeting the anti hypertension activity through molecular docking on endothelin-B receptor domain. Bioinformation 2012;8:81-6.

19. Gaurav A, Gautam V, Pereira S, Alvarez-Leite J, Vetri F, Choudhury M, et al. Structure-based three-dimensional pharmacophores as an alternative to traditional methodologies. J Receptor Ligand Channel Res 2014;7:27-38.

20. Tiwari R, Chakraborty S, Dhama K. Miracle of herbs in antibiotic resistant wounds and skin infections: Treasure of nature-a review/ perspective. Pharm Sci Monit 2013;4:214-48.

21. Mensah AY, Sampson J, Houghton PJ, Hylands PJ, Westbrook J, Dunn M, et al. Effects of Buddleja globosa leaf and its constituents relevant to wound healing. J Ethnopharmacol 2001;77:219-26.

22. Wu F, Bian D, Xia Y, Gong Z, Tan Q, Chen J, et al. Identification of major active ingredients responsible for burn wound healing of Centella asiatica herbs. Evid Based Complement Alternat Med 2012;2012:848093.

23. Martínez-Vázquez M, Ramírez Apan TO, Aguilar H, Bye R. Analgesic and antipyretic activities of an aqueous extract and of the flavone linarin of Buddleia cordata. Planta Med 1996;62:137-40.

24. Martínez-Vázquez M, Ramírez Apan TO, Lastra AL, Bye R. A comparative study of the analgesic and anti-inflammatory activities 
of Pectolinarin isolated from Cirsium subcoriaceum and linarin isolated from Buddleia cordata. Planta Med 1998;64:134-7.

25. Mukherjee PK, Maity N, Nema NK, Sarkar BK. Bioactive compounds from natural resources against skin aging. Phytomedicine 2011;19:64-73

26. Kusano A, Seyama Y, Nagai M, Shibano M, Kusano G. Effects of fukinolic acid and cimicifugic acids from Cimicifuga species on collagenolytic activity. Biol Pharm Bull 2001;24:1198-201.

27. Yoo YC, Yoo SK. The effects of compound madecassol on the wound healing. J Korean Soc Plast Reconstr Surg 1998;25:1451-8.

28. Nafee N, Youssef A, El-Gowelli H, Asem H, Kandil S. Antibiotic-free nanotherapeutics: Hypericin nanoparticles thereof for improved in vitro and in vivo antimicrobial photodynamic therapy and wound healing. Int J Pharm 2013;454:249-58.

29. Lee TH, Lee GW, Park KH, Mohamed MA, Bang MH, Baek YS, et al. The stimulatory effects of Stewartia koreana extract on the proliferation and migration of fibroblasts and the wound healing activity of the extract in mice. Int J Mol Med 2014;34:145-52.

30. Nayak BS, Pinto Pereira LM. Catharanthus roseus flower extract has wound-healing activity in sprague dawley rats. BMC Complement Altern Med 2006;6:41

31. Swaminathan P, Kalva S, Saleena LM. E-pharmacophore and molecular dynamics study of flavonols and dihydroflavonols as inhibitors against dihydroorotate dehydrogenase. Comb Chem High Throughput Screen 2014; 17:663-73

32. Sengupta D, Verma D, Naik PK. Docking mode of delvardine and its analogues into the p66 domain of HIV-1 reverse transcriptase: Screening using molecular mechanics-generalized born/surface area and absorption, distribution, metabolism and excretion properties.
J Biosci 2007;32:1307-16.

33. Schrodinger LL. Small-Molecule Drug Discovery Suite 2013-3: QikProp, version 3.8. New York: Schrodinger, LLC; 2013.

34. Basha SH, Bethapudi P, Majji Rambabu F. Anti-angiogenesis property by Quercetin compound targeting VEGFR2 elucidated in a computational approach. Eur J Biotechnol Biosci 2014;2:30-46.

35. Mohindra JK, Rauth AM. Increased cell killing by metronidazole and nitrofurazone of hypoxic compared to aerobic mammalian cells. Cancer Res 1976;36:930-6.

36. Sulzberger MB, Kanof A, Baer RL, Lowenberg C. Sensitization by topical application of sulfonamides. J Allergy 1947;18:92-103.

37. Varadharaj V, Kandakatl N. Glycogen synthase kinase-3 beta protein inhibition by selected phytocompounds in silico. Asian J Pharm Clin Res 2016;10:87-90.

38. Aparna VA, Mohanalakshmi NA, Dineshkumar KE, Hopper WA. Identification of inhibitors for RND efflux pump of Pseudomonas aeruginosa using structurebased pharmacophore modeling approach. Int J Pharm Pharm Sci 2014;6:84-9.

39. Daisy P, Vijayalakshmi P, Selvaraj C, Singh SK, Saipriya K. Targeting multidrug resistant mycobacterium tuberculosis htrA2 with identical chemical entities of fluoroquinolones. Indian J Pharm Sci 2012;74:217-22

40. Cespedes CL, Sampietro DA, Seigler DS, Rai M, editors. Natural Antioxidants and Biocides from Wild Medicinal Plants. Oxford, UK: CABI; 2013.

41. Han S, Sung KH, Yim D, Lee S, Lee CK, Ha NJ, et al. The effect of linarin on lps-lnduced cytokine production and nitric oxide inhibition in murine macrophages cell line RAW264. 7. Arch Pharm Res 2002;25:170-7. 